\title{
Inseminação artificial intra-uterina em leitoas com sêmen criopreservado com dimetilacetamida e glicerol
}

\author{
Intrauterine artificial insemination of gilts using cryopreserved boar semen with \\ dimetilacetamide and glycerol
}

\author{
Ivan Bianchi' ${ }^{\mathrm{I}}$ Kérlin Calderam ${ }^{\mathrm{I}}$ Éder Francisco Maschio ${ }^{\mathrm{I}}$ Elisângela Mirapalheta Madeira' \\ Rafael da Rosa Ulguim ${ }^{I}$ Gissele Rambo ${ }^{I}$ Érico Kunde Corrêa ${ }^{I}$ Thomaz Lucia Júnior ${ }^{I}$ \\ João Carlos Deschamps ${ }^{\mathrm{I}}$ Marcio Nunes Corrêa ${ }^{\mathrm{II}}$
}

\section{RESUMO}

Este estudo teve por objetivo avaliar o uso da dimetilacetamida (DMA) e de glicerol na criopreservação de sêmen suíno sobre as taxas de concepção e fertilização in vivo, utilizando o método de inseminação artificial pós-cervical. Foram sincronizadas 60 leitoas pré-púberes e inseminadas com o uso de sêmen congelado com glicerol 3\% (30 fêmeas) e DMA 5\% (30 fêmeas). O método de inseminação utilizado foi o pós-cervical, com concentração de $1 \times 10^{9}$ espermatozóides vivos por dose. Após 36 a $40 \mathrm{~h}$ da inseminação, as fêmeas foram abatidas, sendo realizada a contagem de corpos hemorrágicos $(\mathrm{CH})$ nos ovários. Foi realizada a lavagem dos ovidutos das fêmeas, verificando o número de estruturas recuperadas (oócitos e embriões), calculando-se as taxas de concepção e fertilização. A média de $\mathrm{CH}$ nas fêmeas do grupo glicerol 3\% não diferiu $(P>0,05)$ daquelas do grupo DMA 5\% $(10,4 \times 10,2$, respectivamente). Não houve diferença $(P>0,05)$ nas taxas de recuperação de estruturas entre os grupos glicerol $3 \%(68,9 \%)$ e DMA 5\% (66,9\%). Os resultados obtidos nos grupos glicerol 3\% e DMA 5\% para as taxas de concepção (73,3 x 76,6\%) e fertilização (48,6 x 59,4\%) não apresentaram diferença $(P>0,05)$. Conclui-se que não há diferenças nas taxas de concepção e fertilização in vivo utilizando-se sêmen congelado com o uso de dimetilacetamida ou de glicerol.

Palavras-chave: inseminação artificial, congelamento, amidas, glicerol, suínos.

\section{ABSTRACT}

The objective of this study was to evaluate the use of dimethylacetamide (DMA) and glycerol in boar semen cryopreservation on the conception and fertility rates. Sixty pre-pubertal gilts were synchronized and inseminated with semen including either glycerol 3\% or DMA 5\% as cryoprotectants ( $n=30$ for both groups). Artificial insemination was conducted with the intrauterine method using doses with $1 \times 10^{9}$ viable spermatozoa. The gilts were slaughtered 36$40 \mathrm{~h}$ after the insemination and the hemorrhagic bodies $(\mathrm{CH})$ in the ovaries were counted. The oviducts were washed, so oocytes and embryos could be recovered to determine the conception and fertility rates. The average $\mathrm{CH}$ obtained with glycerol 3\% did not differ $(P>0.05)$ from those observed with DMA (10.4 x 10.2, respectively). Recovery rates also did not differ $(P>0.05)$ between groups $(68.9 \%$ with glycerol and $66.9 \%$ with DMA). Conception (73.3 $\times 76.6 \%)$ and fertility rate $(48.6 \times 59.4 \%)$ were also similar $(P>0.05)$ for glycerol and DMA, respectively. Those results indicate that DMA 5\% can replace glycerol $3 \%$ in the composition of extenders for frozen boar semen.

Key words: artificial insemination, cryopreservation, amides, glycerol, swine.

\section{INTRODUÇÃO}

$\mathrm{Na}$ espécie suína, os primeiros animais produzidos por inseminação artificial (IA) com sêmen congelado só foram obtidos em 1970 (POLGE et al., 1970), cerca de 20 anos após a descoberta do efeito crioprotetor do glicerol sobre gametas (POLGE et al., 1949). No entanto, o uso de sêmen suíno congelado determina a queda de 20 a $30 \%$ nos resultados de taxa de parição e 2 a 3 leitões a menos por leitegada (JOHNSON, 1985). Dessa forma, a sua utilização fica condicionada apenas a situações específicas, tais como a produção de reprodutores em núcleos genéticos

${ }^{\mathrm{I} C e n t r o ~ d e ~ B i o t e c n o l o g i a, ~ F a c u l d a d e ~ d e ~ V e t e r i n a ́ r i a ~(F A V E T), ~ U n i v e r s i d a d e ~ F e d e r a l ~ d e ~ P e l o t a s ~(U F P e l), ~ P e l o t a s, ~ R S, ~ B r a s i l . ~}$

IIDepartamento de Clínica Veterinária, Núcleo de Pesquisa, Ensino e Extensão em Pecuária (NUPEEC), FAVET, UFPel, Campus

Universitário, s/n, 96010 900, Pelotas, RS, Brasil. E-mail: marcio.nunescorrea@pesquisador.cnpq.br. Autor para correspondência. 
(CRABO, 1990). A grande sensibilidade do espermatozóide (SPTZ) ao choque térmico é o que impede a utilização em maior escala do sêmen congelado nesta espécie.

O glicerol é normalmente o crioprotetor de eleição para o congelamento de sêmen na maioria das espécies de mamíferos. Estudos com a utilização de crioprotetores em substituição ou associação com o glicerol têm sido conduzidos especialmente em eqüinos com o uso de diferentes amidas (ALVARENGA et al., 2000; GRAHAN, 2000; MEDEIROS et al., 2002; VIDAMENT et al., 2002). Amidas também são utilizadas nos protocolos de congelamento de sêmen de aves (LUKASZEWICZ et al., 2004) e de peixes (OGIER DE BAULNY et al., 1999). A superioridade do uso de dimetilacetamida em relação ao glicerol em avaliação in vitro de sêmen suíno congelado foi demonstrada por BIANCHI et al. (2006a; 2006b).

No emprego de sêmen congelado através da IA intracervical, normalmente são utilizadas doses de 5-6 x 109 SPTZ (BORDIGNON et al., 1996; ROCA et al., 2003), o que é consideravelmente superior à dose convencional de $3 \times 10^{9}$ SPTZ utilizada com sêmen refrigerado (SERRET et al., 2005). Porém, alguns trabalhos têm apresentado resultados promissores de fertilidade utilizando sêmen suíno congelado através da IA intracervical (ERIKSSON et al., 2002; ROCA et al., 2003; ROCA et al., 2006). A melhora nos resultados obtidos é resultante da adaptação de protocolos de criopreservação, além do desenvolvimento de novos métodos de IA, tais como o intra-uterino profundo, utilizando doses de até $150 \times 10^{6} \mathrm{SPTZ}$. Outro método de IA que tem sido testado, especialmente com o emprego de sêmen refrigerado, é o pós-cervical (WATSON \& BEHAN, 2002; BENNEMANN et al., 2004; SERRET et al., 2005), através do qual tem se alcançado sucesso com emprego de doses de $1 \times 10^{9}$ SPTZ. MARTINEZ et al., (2006), ao compar IA intrauterina profunda em relação ao método convencional intracervical, encontraram $21 \%$ de fertilizações unilaterais, associadas ao método intra-uterina profundo, ainda que tivessem utilizado sêmen refrigerado.

O objetivo deste estudo foi avaliar o uso da dimetilacetamida (DMA) e glicerol na criopreservação de sêmen suíno sobre as taxas de concepção e fertilização in vivo, utilizando o método de inseminação artificial pós-cervical.

\section{MATERIAL E MÉTODOS}

Foi utilizado sêmen de um macho suíno cruzado (Landrace x Large White), com aproximadamente 24 meses de idade, de fertilidade conhecida, em regime de coleta. $\mathrm{O}$ animal foi mantido em baia individual, na Estação Experimental da Palma (Universidade Federal de Pelotas-RS). O ejaculado foi coletado por meio do método da mão-enluvada (BEARDEN e FUQUAY, 1997a), usando um copo plástico protegido por um copo isotérmico recoberto por gaze, a fim de separar a fração do ejaculado rica em gel, para depois descartar esta fração. A condição mínima para o ejaculado ser processado foi de motilidade espermática e integridade de membrana por fluorescência superior a $80 \%$ e $70 \%$, respectivamente, no momento da coleta.

Imediatamente após a coleta do sêmen, a fração rica em espermatozóides (SPTZ) foi diluída (1:1, v/v) em tubo em BTS (LEVIS, 2000). O método de congelamento utilizado foi o descrito por WESTENDORF etal.(1975) e BORDIGNON etal.(1996), com modificações realizadas por BIANCHI et al. (2006a, 2006b). A curva de resfriamento após a diluição inicial foi de $60 \mathrm{~min}$ a $24^{\circ} \mathrm{C}$, sendo que após este período as amostras foram mantidas por $60 \mathrm{~min} \mathrm{a} 15^{\circ} \mathrm{C}$. Ao atingir $15^{\circ} \mathrm{C}$, foi realizada a centrifugação $(800 \mathrm{x} g$ por $10 \mathrm{~min})$, para a retirada do plasma seminal. O sedimento de espermatozóides (SPTZ) obtido da centrifugação foi resuspenso no diluidor de resfriamento (DR) GEMA $(80 \%, v / v$, de solução de lactose a $11 \% ; 20 \%$ gema de ovo, v/v), num volume suficiente para que cada $\mathrm{mL}$ mantivesse uma concentração de $450 \times 10^{6}$ de SPTZ. Depois foi realizado o resfriamento por $90 \mathrm{~min}$ até $5^{\circ} \mathrm{C}$, quando foi resuspenso nos diluidores de congelamento (DC) à base dos crioprotetores Glicerol $\left(\mathrm{C}_{3} \mathrm{H}_{8} \mathrm{O}_{3}\right)$ e N,NDimetilacetamida $\left(\mathrm{C}_{4} \mathrm{H}_{9} \mathrm{NO}\right)$, com pesos moleculares de 92,09 e 87,12, respectivamente.

Os DC foram elaborados a partir do DR, sendo o DC composto de glicerol (89,5\% de DR GEMA $+1,5 \%$ Orvus Ex Paste, Equex-Paste e 9\% de Glicerol, v/v), e o DC dimetilacetamida (83,5\% de DR GEMA + 1,5\% Orvus Ex Paste, Equex-Paste e 15\% de DMA, v/ v).

A quantidade de DC acrescentada foi de 1:2, ou seja, para cada $2 \mathrm{~mL}$ de sêmen com $\mathrm{DR}$, foi adicionado $1 \mathrm{~mL}$ de DC. Nesta proporção obteve-se uma concentração final de 3\% para o congelamento com glicerol (WESTENDORF et al., 1975) e a 5\% para DMA (BIANCHI et al., 2006a; 2006b). Depois da adição do DC, o sêmen foi envasado em palhetas de $0,5 \mathrm{~mL}$, contendo a concentração de $150 \times 10^{6} \mathrm{SPTZ} /$ palheta. As palhetas foram congeladas horizontalmente em vapor de nitrogênio líquido, $5 \mathrm{~cm}$ acima do nível do nitrogênio, a uma temperatura aproximada de $-90^{\circ} \mathrm{C}$ por um período de $20 \mathrm{~min}$, sendo após mergulhadas em nitrogênio líquido a $-196^{\circ} \mathrm{C}$ e mantidas até o descongelamento. 
As palhetas foram descongeladas a $37^{\circ} \mathrm{C}$ por 20s, sendo resuspensas em tubo cônico contendo $10 \mathrm{~mL}$ de BTS previamente aquecido a $37^{\circ} \mathrm{C}(1: 20, \mathrm{v} / \mathrm{v})$.

Após o descongelamento, foi feita a incubação em banho-maria a $37^{\circ} \mathrm{C}$ por 10 min e avaliada a motilidade ( 0 a $100 \%)$ por meio de microscopia ótica com contraste de fases em aumento de 200 vezes (BEARDEN e FUQUAY, 1997b).

Após a avaliação da motilidade e vigor, foi realizada a avaliação da integridade de membrana espermática por fluorescência (HARRISON e VICKERS, 1990), através das sondas Diacetato de Carboxifluoresceína (CFDA) e Iodeto de Propídio (IP). Foi preparado $1 \mathrm{~mL}$ de uma solução contendo $950 \mu \mathrm{L}$ de citrato de sódio $3 \%, 20 \mu \mathrm{L}$ de IP, $20 \mu \mathrm{L}$ de CFDA e $10 \mu \mathrm{L}$ de formoldeído $40 \%$ (Solução 1). Em seguida, $40 \mu \mathrm{L}$ da Solução 1 e $10 \mu \mathrm{L}$ de amostra do sêmen descongelado foram colocadas em ependorf de $250 \mu \mathrm{L}$ (Solução 2). O tubo foi guardado em sala escura em temperatura de $22^{\circ} \mathrm{C}$ por 15 min, quando então $5 \mu \mathrm{L}$ da Solução 2 foi colocada sobre uma lâmina com lamínula e avaliada em microscópio de epifluorescência (Olympus BX 51, América INC), por meio de excitação em filtro WU sob aumento de 400x. Foram contados 200 espermatozóides em uma mesma lâmina e classificados conforme sua coloração em íntegros (corados em verde em toda sua extensão) e lesados (corados em vermelho).

A integridade funcional da membrana espermática foi testada também por meio do teste do choque hipoosmótico (CHIPO) (VASQUEZ et al., 1997). A avaliação foi feita com uma câmara de Neubauer e microscopia óptica com contraste de fases, em aumento de 400 vezes, procedendo-se à contagem de 100 células, registrando o número de células que apresentavam cauda do espermatozóide normal e aquelas que estavam dobradas ou enroladas. $\mathrm{O}$ valor que foi utilizado para análise do CHIPO correspondeu à diferença obtida entre o número de espermatozóides com cauda dobrada e enrolada, observadas no teste realizado com a solução hiposmótica $\left(0 \mathrm{mOsm} \mathrm{kg}^{-1}\right)$ e isosmótica $\left(312 \mathrm{mOsm} \mathrm{kg}^{-1}\right)$.

Para avaliação da capacidade fertilizante in vivo do sêmen congelado em glicerol e dimetilacetamida, 60 leitoas pré-púberes (30 para cada grupo) com peso médio de $86,5 \mathrm{~kg}$ e idade de $155 \mathrm{~d}$ foram tratadas com $1000 \mathrm{UI}$ de eCG (sendo considerado hora 0 ) e 70-72 h após com de 500 UI de hCG (CORRÊA et al., 2006). Ambos os hormônios foram administrados por via intramuscular. Entre 31-34h após a aplicação do $\mathrm{hCG}$, as leitoas foram inseminadas ao acaso, independente dos sinais de cio e abatidas entre 36 a 40h após a IA (BORDIGNON et al., 1996).

Para elaboração das doses inseminantes, foi realizado o descongelamento das palhetas do tratamento correspondente (glicerol ou DMA) em banho-maria a $37^{\circ} \mathrm{C}$ por 20 s imediatamente antes de cada IA. Após o descongelamento, o conteúdo das palhetas foi adicionado em uma dose de $60 \mathrm{~mL}$ de BTS em temperatura de $37^{\circ} \mathrm{C}$ (BORDIGNON et al., 1996), a fim de atingir uma concentração de $1 \times 10^{9} \mathrm{SPTZ}$ vivos (baseados na análise prévia da motilidade espermática).

O método de IA utilizado foi o pós-cervical, em que a pipeta é fixada na cérvix e possui um cateter com um prolongamento de $20 \mathrm{~cm}$, o que permite a deposição do sêmen no corpo do útero (WATSON \& BEHAN, 2002; SERRET et al., 2005).

No frigorífico as genitálias das fêmeas foram coletadas e transportadas para o laboratório, onde foi determinada a taxa de ovulação para cada fêmea, a partir da contagem do número de corpos hemorrágicos em cada ovário. Para a colheita das estruturas (oócitos e embriões), cada oviduto (direito e esquerdo) foi lavado com $20 \mathrm{~mL}$ de PBS (solução de tampão fosfato) no sentido útero-ovário, sendo que o conteúdo foi lavado dentro de uma placa de petri e foi realizada a procura em lupa.

Para cada fêmea, foram determinadas as taxas de recuperação (TR) e fertilização (TF), a partir das seguintes fórmulas: Taxa de recuperação (TR\%); $\mathrm{TR}=\left(\mathrm{n}^{\mathrm{o}}\right.$ oócitos e/ou embriões recuperados / $\mathrm{n}^{\mathrm{o}}$ corpos hemorrágicos contados) x 100; Taxa de fertilização (TF\%); $\mathrm{TF}=\left(\mathrm{n}^{\circ}\right.$ de embriões / no de estruturas recuperadas) x 100. A taxa de concepção foi determinada considerando o número de fêmeas em que foi verificada a presença de embriões em relação ao total de fêmeas inseminadas.

Estatísticas descritivas foram calculadas para as avaliações espermáticas in vitro, o número de corpos hemorrágicos, oócitos e embriões e a taxa de recuperação e fertilização. A análise de concepção entre os grupos glicerol e DMA foi avaliada pelo teste do qui-quadrado. As variáveis dependentes consideradas na análise de variância foram: número de corpos hemorrágicos, oócitos, embriões, taxas de recuperação e fertilização. A variável independente considerada foi o crioprotetor utilizado (glicerol ou DMA). A comparação de médias foi feita pelo teste de Tukey. Todas as análises foram realizadas por meio do STATISTIX $^{\circledR}$ (2004).

\section{RESULTADOS E DISCUSSÃO}

Os valores de $\mathrm{pH}$ e osmolalidade do sêmen e dos diluidores para resfriamento e congelamento do sêmen estão apresentados na tabela 1. Os resultados de avaliação in vitro do sêmen após a coleta e após o descongelamento estão apresentados na tabela 2 . A sensibilidade do espermatozóide suíno ao processo 
Tabela 1 - Valores de pH e osmolalidade do sêmen e diluidores utilizados.

\begin{tabular}{|c|c|c|}
\hline \multirow{2}{*}{ Item avaliado } & \multirow[b]{2}{*}{$\mathrm{pH}$} & \multirow[b]{2}{*}{ Osmolalidade, mOsmol kg-1 $\mathrm{H}_{2} \mathrm{O}$} \\
\hline & & \\
\hline Sêmen & 7,5 & 302 \\
\hline BTS & 6,9 & 332 \\
\hline Diluidor de resfriamento Gema de Ovo & 6,1 & 351 \\
\hline Diluidor de congelamento Glicerol & 6,7 & 1.990 \\
\hline Diluidor de congelamento DMA & 6,7 & 3.343 \\
\hline
\end{tabular}

de congelamento-descongelamento é evidenciada na comparação dos resultados das avaliações do sêmen após a coleta, com os resultados obtidos após o congelamento. Na comparação entre os crioprotetores glicerol e DMA nas avaliações após o descongelamento, evidencia-se a superioridade dos resultados de motilidade e integridade de membrana por fluorescência obtidos no tratamento com DMA. Para a elaboração das doses inseminantes, foi utilizado o resultado de motilidade obtido após o descongelamento (Tabela 2), calculando-se dessa forma a quantidade de palhetas utilizadas a fim de atingir $1 \times 10^{9} \mathrm{SPTZ}$ vivos por dose.

Em todas as fêmeas utilizadas no trabalho, foram encontrados nos ovários folículos préovulatórios e/ou corpos hemorrágicos, demonstrando que todas responderam ao protocolo hormonal utilizado. O número de corpos hemorrágicos, a taxa de recuperação e os resultados de concepção e fertilização para cada grupo estão apresentados na tabela 3. A média de corpos hemorrágicos foi superior àquelas obtidas por CORRÊA et al. (2006), porém, inferiores às médias obtidas por BORDIGNON et al. (1996), enquanto que as taxas de recuperação obtidas se assemelham aos dados desses autores. Este trabalho foi realizado com o uso de leitoas pré-púberes, que, embora tenham respondido ao protocolo hormonal, a taxa de ovulação é baixa quando comparado ao uso de fêmeas multíparas (ROCA et al., 2003).
Usando sêmen congelado, BORDIGNON et al. (1996) conseguiram $85 \%$ de taxa de concepção trabalhando com leitoas pré-púberes sincronizadas. No entanto, esses autores utilizaram IA intracervical com doses de $6 \times 10^{9}$ SPTZ. ROCA et al. (2003) obtiveram em torno de $79 \%$ de taxa de prenhez aos $28 \mathrm{~d}$, com o emprego de sêmen congelado por meio do método de IA intra-uterina profunda com dose de $1 \times 10^{9} \mathrm{SPTZ}$ e na IA intracervical com doses com $6 \times 10^{9} \mathrm{SPTZ}$, porém, eles utilizaram fêmeas multíparas. ERIKSSON et al. (2002) utilizando IA intracervical com sêmen congelado em fêmeas multíparas, alcançaram $72 \%$ de taxa de parto, usando protocolo com duas inseminações por estro, com concentração de $5 \times 10^{9} \mathrm{SPTZ}$ por dose. Taxa de prenhez de $40 \%$ aos 28 é citada com o uso de IA intrauterina profunda utilizando dose de $1 \times 10^{9}$ de SPTZ (WONGTAWAN et al., 2006).

Não houve diferença entre os grupos glicerol e DMA na taxa de concepção $(\mathrm{P}>0,05)$ e os resultados se assemelharam aos relatados com o uso de sêmen congelado. A taxa de fertilização do grupo glicerol é comparável à obtida por BORDIGNON et al. (1996), porém, a fertilização do grupo DMA é superior aos resultados desses autores. No entanto, os resultados neste estudo são oriundos de IA pós-cervical com doses de 1 X $10^{9}$ de SPTZ, ao contrário das doses de 5-6 X $10^{9}$ de SPTZ, embora utilizando IA intracervical. Além disso, destacam-se os resultados obtidos com o uso de DMA, pois a maioria dos trabalhos que utilizam sêmen suínos congelado baseia-se no uso de glicerol.

Tabela 2 - Parâmetros de avaliação do sêmen após a coleta e após o congelamento.

\begin{tabular}{lcc}
\hline & & \\
Parâmetro avaliado & Pós-coleta & Glicerol \\
& & 53,0 \\
\hline Motilidade, \% & 85,0 & 45,0 \\
Integridade de membrana por fluorescência, \% & 77,0 & 65,0 \\
Integridade de membrana pelo CHIPO $\%$ DMA $\%$ & 35,0 & 26,0 \\
\hline
\end{tabular}

*CHIPO: Choque hipoosmótico.

Ciência Rural, v.38, n.7, out, 2008. 
Tabela 3 - Taxa de recuperação de estruturas e efeito do tratamento sobre a fertilidade.

\begin{tabular}{lcc}
\hline & \multicolumn{2}{c}{--------Tratamento--------- } \\
& Glicerol 3\% & DMA 5\% \\
\hline Leitoas inseminadas & 30 & 30 \\
Corpos hemorrágicos & 306 & 299 \\
Taxa de recuperação, \% & 68,9 & 66,9 \\
Taxa de concepção, \% & 73,3 & 76,6 \\
Taxa de fertilização, \% & 48,6 & 59,4 \\
\hline
\end{tabular}

Os dados não diferem $(\mathrm{P}>0,05)$.

Não foi encontrada diferença $(\mathrm{P}>0,05)$ na taxa de fertilização entre os grupos glicerol e DMA. No entanto, o grupo DMA é superior $10 \%$ em relação ao glicerol, além do que possui uma tendência de maior taxa de concepção. Além disso, os resultados da avaliação espermática pós-descongelamento devem ser enfatizados. Com base nas avaliações de motilidade para elaborar as doses inseminantes, foram necessárias 10 palhetas do tratamento DMA, enquanto que para atingir a mesma concentração foram utilizadas 13 palhetas de glicerol. Essa diferença de 30\% torna o processo com o uso de DMA mais eficiente, resultando em um melhor aproveitamento do ejaculado.

Devido ao fato do uso de IA com sêmen congelado, especial atenção deve ser dada ao momento da IA, pois há um menor período necessário para a capacitação dos espermatozóides, além do menor tempo de permanência destes no oviduto (JOHNSON, 1985; ROCA et al., 2003). Com o protocolo hormonal utilizado, a estimativa é de que os animais ovularam $42 \pm 2 \mathrm{~h}$ após a aplicação do hCG (SRIKANDAKUMAR \& DOWNEY, 1989; HUHN et al., 1996). A taxa de fertilização com sêmen descongelado pode diminuir quando as fêmeas são inseminadas mais que quatro horas antes ou após a ovulação (WABERSKI et al., 1994). No entanto, não foi feito o acompanhamento da dinâmica folicular. Dessa forma, o momento da IA pode ter influenciado especialmente a taxa de fertilização, e melhores resultados poderão ser obtidos com a IA realizada o mais próximo possível do momento da ovulação, com acompanhamento da ultra-sonografia.

Os resultados deste estudo sugerem que a DMA pode ser utilizada como substituto ao glicerol em protocolos para congelamento de sêmen suíno.

\section{CONCLUSÕES}

A inseminação de fêmeas suínas a partir do método pós-cervical utilizando sêmen congelado com o uso de dimetilacetamida ou de glicerol não alterou as taxas de concepção e fertilização in vivo.

\section{AGRADECIMENTO}

Este estudo foi apoiado pela Coordenação de Aperfeiçoamento de Pessoal de Nível superior (CAPES) e pelo Conselho Nacional de Desenvolvimento Científico e Tecnológico (CNPq).

\section{COMITÊ DE ÉTICA E BIOSSEGURANÇA}

Este estudo foi aprovado pelo comitê de ética e biossegurança da UFPel (Protocolo 011-2006).

\section{REFERÊNCIAS}

ALVARENGA, M.A. et al. Alternative cryoprotectors for freezing stallion spermatozoa. In: INTERNATIONAL CONGRESS ON ANIMAL REPRODUCTION AND ARTIFICIAL INSEMINATION, 14., 2000, Stockholm. Proceedings... Stockholm, 2000. p.157.

BEARDEN, H.J.; FUQUAY, J.W. Semen collection. In: Applied animal reproduction. 4.ed. New Jersey: Prentice Hall, 1997a. Cap.14, p.147-157.

BEARDEN, H.J.; FUQUAY, J.W. Semen evaluation. In: Applied animal reproduction. 4.ed. New Jersey: Prentice Hall, 1997b. Cap.15, p.159-170.

BENNEMANN, P.E. et al. Reproductive performance of sows submitted to intrauterine insemination at different preovulatory intervals. Animal Reproduction, v.1, p.106-110, 2004.

BIANCHI, I. et al. Uso de diferentes crioprotetores no congelamento de sêmen suíno. In: CONGRESSO LATINOAMERICANO DE SUINOCULTURA, 3., 2006, Foz do Iguaçu. Anais... Concórdia: Embrapa Suínos e Aves, 2006a, CD-ROM.

BIANCHI, I. et al. Congelamento de sêmen suíno usando amidas em diferentes concentrações. In: CONGRESSO LATINOAMERICANO DE SUINOCULTURA, 3., 2006, Foz do Iguaçu. Anais... Concórdia: Embrapa Suínos e Aves, 2006b. CDROM.

BORDIGNON, V. et al. Effect of trehalose on motility, acrosome and fertility of the frozen-thawed boar semen. Revista Brasileira de Reprodução Animal, v.20, p.54-62, 1996.

CORRÊA, M.N. et al. Swine semen cooled at $5{ }^{\circ} \mathrm{C}$ with PIGPEL5 extender: effects on parameters of semen quality in vitro and fertility estimators in vivo. Animal Reproduction, v.3, p.4148,2006

CRABO, B.G. Preservation of boar semen: a worldwide perspective. In: INTERNATIONAL CONFERENCE BOAR SEMEN PRESERVATION, 2., 1990, Beltsville. Proceedings... Beltsville: 1990. p.3-9.

ERIKSSON, B.M. et al. Field fertility with exported boar semen frozen in the new flat-pack container. Theriogenology, v.58, p.1065-1079, 2002.

Ciência Rural, v.38, n.7, out, 2008. 
GRAHAM, J.K. Evaluation of alternative cryoprotectants for preserving stallion spermatozoa. In: INTERNATIONAL CONGRESS ON ANIMAL REPRODUCTION AND ARTIFICIAL INSEMINATION, 14., 2000, Stockholm. Proceedings... Stockholm, 2000. p.307.

HARRISON, R.A.P.; VICKERS, S.E. Use of fluorescent probes to assess membrane integrity in mammalian spermatozoa. Journal Reproductive and Fertility. 88, p.343-352, 1990.

HUHN, P.E. et al. Techniques developed for the control of estrus ovulation and parturition in the east german pig industry: A review. Theriogenology, v.46, p.911-924, 1996

JOHNSON, L.A. Fertility results using frozen boar spermatozoa 1970 to 1985 . In: INTERNATIONAL CONFERENCE BOAR SEMEN PRESERVATION, 1985, Uppsala. Proceedings... Uppsala, 1985. p.199-222.

LEVIS, D.G. Liquid boar semen production: current extender technology and where do we go from here! In INTERNATIONAL CONFERENCE ON BOAR SEMEN PRESERVATION, 4., 2000, Beltsville. Proceedings... Beltsville, 2000. p.121-128.

LUKASZEWICZ, E. et al. Attempts on freezing the Greylag (Anser anser L.) gander semen. Animal Reproduction Science, v.80, p.163-173, 2004.

MARTINEZ, E.A. et al. Incidence of unilateral Fertilizations after low dose deep intrauterine insemination in spontaneously ovulating sows under field conditions. Reproductive in Domestic Animals, v.41, p.41-47, 2006.

MEDEIROS, A.S.L. et al. Cryopreservation of stallion sperm using different amides. Theriogenology, v.58, p.273-276, 2002 .

OGIER DE BAULNY, B. et al. Membrane integrity, mitochondrial activity, ATP content and motility of European Catfish (Silurus glanis) testicular spermatozoa after freezing with different cryoprotectants. Cryobiology, v.39, p.177$184,1999$.

POLGE, C. et al. Fertilizing capacity of frozen boar semen following surgical insemination. Veterinary Record, v.87, p.424-429, 1970.
POLGE, C. et al. Revival of spermatozoa after vitrification and dehydration at low temperature. Nature, p.164-166, 1949.

ROCA, J. et al. Factors influencing boar sperm cryosurvival. Journal of Animal Science, v.84, p.2692-2699, 2006.

ROCA, J. et al. Fertility of weaned sows after deep intrauterine insemination with a reduced number of frozen-thawed spermatozoa. Theriogenology, v.60, p.77-87, 2003.

SERRET, C.G. et al. Intrauterine artificial insemination in female swine with distinct sperm concentrations, parities and methods of ovulation estimation. Animal Reproduction, v.2, p.250-256, 2005 .

SRIKANDAKUMAR, A.; DOWNEY, B.R. Induction of ovulation in gilts with cloprostenol. Theriogenology, v.32, p.445-449, 1989 .

STATISTIX ${ }^{\circledR}$. Statistix for Windows user's manual. Ed. Analytical Software. Tallahassee, Fl, 2004.

VASQUEZ, J.M. et al. Hypoosmotic swelling of boar spermatozoa compared to other methods for analysing the sperm membrane. Theriogenology, v.47, p.913-922, 1997.

VIDAMENT, M. et al. Motility and fertility of stallion semen frozen with glycerol and/or dimethyl formamide. Theriogenology, v.58, p.249-251, 2002.

WABERSKI, D. et al. Effect of time of insemination relative to ovulation on fertility with liquid and frozen boar semen. Theriogenology, v.42, p.831-840, 1994.

WONGTAWAN, T. et al. Fertility after deep intra-uterine artificial insemination of concentrated low-volume boar semen doses. Theriogenology, v.65, p.773-787, 2006.

WATSON, P.F.; BEHAN, J.R. Intrauterine insemination of sows with reduced sperm numbers: results of a commerciallybased field trial. Theriogenology, v.57, p.1683-1693, 2002.

WESTENDORF, P. et al. Zur Tiefgefrierung von Ebersperma: Labor - und besamungsergebnisse mit dem hülsenberger paillettenverfähren. Deutsche Tierarztliche Wochenschrift, v.82, p. 261-267,1975. 